\title{
Artificial Emotion Generation Based on Personality, Mood, and Emotion for Life-Like Facial Expressions of Robots
}

\author{
Jeong Woo Park, Woo Hyun Kim, Won Hyong Lee, and Myung Jin Chung \\ Department of Electrical Engineering, KAIST, Daejeon, Korea \\ \{pjw, ishsrain, leestation\}@errlab.kaist.ac.kr, \\ mjchung@ee.kaist.ac.kr
}

\begin{abstract}
We can't overemphasize the importance of robot's emotional expressions as robots step into human's daily lives. So, the believable and socially acceptable emotional expressions of robots are essential. For such human-like emotional expression, we have proposed an emotion generation model considering personality, mood and history of robot's emotion. The personality module is based on the Big Five Model (OCEAN Model, Five Factor Model); the mood module has one dimension such as good or bad, and the emotion module uses the six basic emotions as defined by Ekman. Unlike most of the previous studies, the proposed emotion generation model was integrated with the Linear Dynamic Affect Expression Model (LDAEM), which is an emotional expression model that can make facial expressions similar to those of humans. So, both the emotional state and expression of robots can be changed dynamically.
\end{abstract}

Keywords: Artificial emotion, Human-like emotional expression, Integration of emotion generation model and expression model.

\section{Introduction}

As robots step into the human's daily lives, we can't stress enough the importance of natural human-robot interaction. Especially, emotional expression is an indispensable part of that natural interaction.

Many previous studies about generation and expression of artificial emotion have been conducted so far. In Kismet's emotional architecture, emotion is a medium to construct a kind of social relationship through emotional expression [1]. Kshirsagar et al. used the 'Multilayer Personality Model' to implement a virtual agent with artificial emotion [2]. A. Egges proposed the PME model to generate artificial emotion of conversational agents [3]. J. C. Park proposed a method to generate emotions using a two-layered architecture that consists of a reactive layer and a deliberative layer [4]. Sajal Chandra Banik suggested a Markovian emotion model using a conditional probability at each emotional state [5]. H. Miwa mentioned emotion dynamics using second order differential equations [6].

However, there have been few studies about the dynamics of facial expressions. In some of the previous studies, we can observe that facial expressions are changed with 
dynamics, but it is simply caused by motor dynamics. Furthermore, there were also very few studies about unified emotion generation and the expression system. Most of the previous studies have handled only the emotion generation model. So, in this paper, we will propose an integrated architecture of emotion generation and an expression system that has emotional dynamics.

\section{Overall Architecture of Emotion Generation and Expression Model}

Our model consists of two parts. The first is an emotion generation model and the second is an emotion expression model. The former is based on the PME (Personality Mood Emotion) model [3] and the latter is based on the LDAEM (Linear Dynamic Affect Expression Model) [10]. New emotion ${ }^{\mathrm{d}} \mathbf{e}$ is generated using user's input and personality as an external input. In addition to the external inputs, previous emotion and current mood are considered internally. The generated emotion is transferred to the emotion expression module, LDAEM, which can obtain a trajectory of all action units from current expression to the next expression.

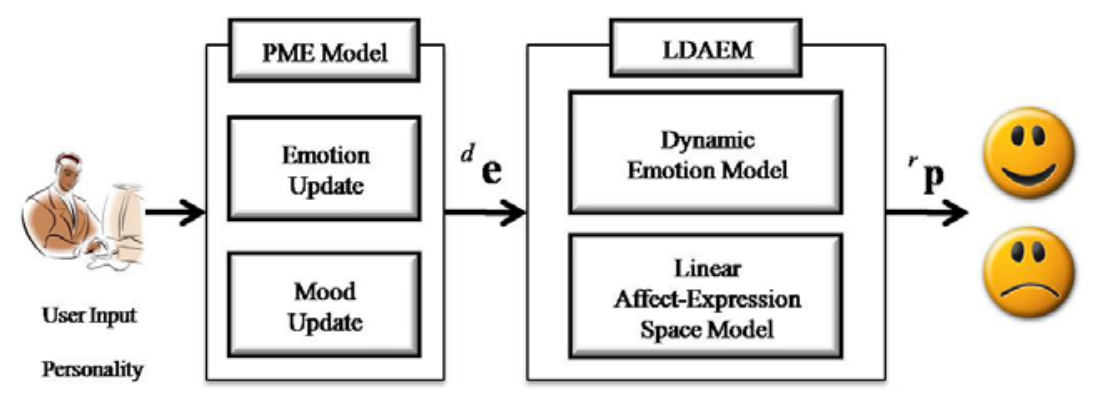

Fig. 1. Overall architecture of emotion generation and expression model

\section{Emotion Generation Model}

\subsection{PME Model}

Our proposed emotion generation model is based on the PME (Personality Mood Emotion) model [3]. The PME model is a quite excellent basic framework for generating artificial emotions. This basic framework consists of a mood updating step and an emotion updating step, as shown in (1) and (2). In the mood updating step, the previous mood, $\mathbf{m}_{t}$, an amount of mood change by input $\Psi_{\mathrm{m}}(\cdot)$, and the internal updating by itself $\Omega_{\mathrm{m}}(\cdot)$ are considered to generate the new mood $\mathbf{m}_{t+l}$. In the emotion updating step, the previous emotion $\mathrm{e}_{t}$, an amount of emotion change by input $\Psi_{\mathrm{e}}(\cdot)$, and the internal updating by constant term $\Omega_{\mathrm{e}}(\cdot)$ are considered to generate the new emotion $\mathbf{e}_{t+l}$. 


$$
\begin{aligned}
\mathbf{m}_{t+1} & =\mathbf{m}_{t}+\Psi_{m}(\cdot)+\Omega_{m}(\cdot) \\
\mathbf{e}_{t+1} & =\mathbf{e}_{t}+\Psi_{e}(\cdot)+\Omega_{e}(\cdot)
\end{aligned}
$$

So, we can make various emotion generation models by using this framework with other kinds of mood and emotion updating functions.

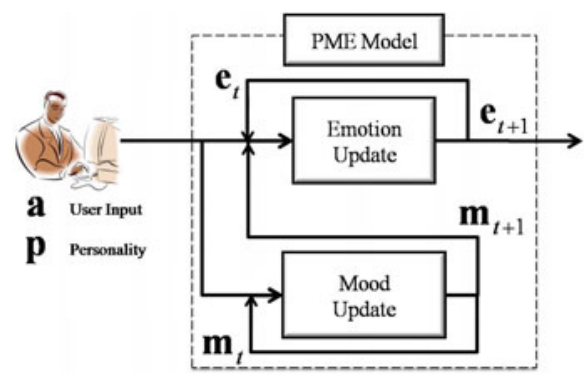

Fig. 2. Block diagram of PME Model

\subsection{Proposed Emotion Generation Model}

In this paper, we used a five dimensional personality based on the Big Five Model [7], a one dimensional mood (good-bad), and a six dimensional emotion based on Ekman's six basic emotions [8].

According to A. Egges and S. Kshirsagar, the characteristics of personality, mood, and emotion are as listed in Fig. 3.

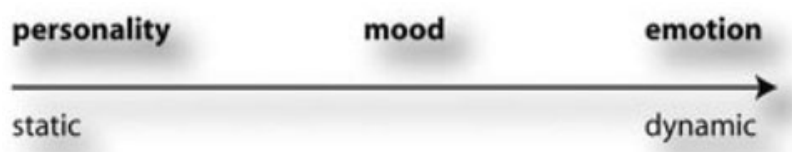

Fig. 3. Characteristics of personality, mood, and emotion [3]

However, mood state in their results is as dynamic as emotion state, because they considered only current input stimuli in the function $\Psi_{\mathrm{m}}(\cdot)$. Namely, the mood state is varied immediately by input stimuli.

Thus, we used some functions different from those used in the previous PME model to update mood and emotion. First, we considered not only current input stimuli but also the history of input stimuli and emotions to update mood, as shown in (3).

$$
\Psi_{m}(\cdot)=R \cdot Q \cdot \mathbf{a} \rightarrow \Psi_{m}(\cdot)=R \cdot Q \cdot\left(\boldsymbol{\gamma}_{t}+\boldsymbol{\omega}_{t}\right)
$$


Here, $Q$ is an emotion-mood influence matrix that defines the relation between the emotions and each mood dimension, $\mathbf{a}$ is a vector of user inputs, $\gamma_{t}$ is a history of user inputs, and $\omega_{\mathrm{t}}$ is a history of emotions. Both $\gamma_{\mathrm{t}}$ and $\omega_{\mathrm{t}}$ can be obtained as follows:

$$
\begin{aligned}
& \boldsymbol{\gamma}_{t}=\frac{1}{s} \sum_{i=0}^{s}\left(\mathbf{a}_{i} \frac{\exp (i)-1}{\exp (s)-1}\right)_{i}, \boldsymbol{\omega}_{t}=\frac{1}{p} \sum_{i=0}^{p}\left(\mathbf{e}_{i} \frac{\exp (i)-1}{\exp (p)-1}\right) \\
& \mathbf{a}_{i}=\left[\begin{array}{lll}
a_{1} & \cdots & a_{l}
\end{array}\right]^{T}, \quad l: \text { number of input stimuli } \\
& \mathbf{e}_{i}=\left[\begin{array}{lll}
e_{1} & \cdots & e_{m}
\end{array}\right]^{T}, \quad m: \text { dimension of emotion, } m=l \\
& s: \text { number of used input stimuli in a history } \\
& p: \text { number of used emotions in a history }
\end{aligned}
$$

We can make a diagonal matrix $R$ using vector $\mathbf{v}$. This vector $\mathbf{v}$ indicates the stability of each mood. In this paper, vector $\mathbf{v}$ is a scalar because we used only one dimensional mood. So, if this value is too big, the mood state will be unstable.

$$
\begin{aligned}
& R=\operatorname{diag}\left(\kappa_{1}, \cdots, \kappa_{k}\right) \\
& \mathbf{v}=R_{0} \cdot \mathbf{p}=\left[\begin{array}{lll}
\kappa_{1} & \cdots & \kappa_{k}
\end{array}\right]^{T}, k: \text { dimension of mood } \\
& \mathbf{p}=\left[\begin{array}{lll}
p_{1} & \cdots & p_{n}
\end{array}\right]^{T}, n: \text { dimension of personality }
\end{aligned}
$$

Here, $R_{0}$ is a personality-mood influence matrix that indicates how each personality factor influences each mood dimension.

In the internal updating part of mood, we simply used the same self-decay process that was used in the previous PME model.

$$
\Omega_{m}(\cdot)=-C_{m} \cdot \mathbf{m}_{t}, C_{m}: \text { constant }
$$

As can be seen in the above equation, we used a history of inputs and emotions to update mood. This was to implement a general property of mood, which is the fact that mood is less dynamic than emotion.

In the part of the emotion updated by input stimuli, the same function in the previous PME model was used.

$$
\Psi_{e}(\cdot)=P \cdot \mathbf{a}+\left(V \cdot \mathbf{m}_{t+1}\right)^{T} \cdot \mathbf{a}
$$

Here, $V$ is a mood-emotion influence matrix that defines the relation between the mood and each emotion.

We can construct a diagonal matrix $P$ using vector $\mathbf{u}$. This vector $\mathbf{u}$ means the importance of each emotion depending on the personality.

$$
\begin{aligned}
& P=\operatorname{diag}\left(\kappa_{1}, \cdots, \kappa_{k}\right) \\
& \mathbf{u}=P_{0} \cdot \mathbf{p}=\left[\begin{array}{lll}
\varepsilon_{1} & \cdots & \varepsilon_{m}
\end{array}\right]^{T}, m: \text { dimension of emotion } \\
& \mathbf{p}=\left[\begin{array}{lll}
p_{1} & \cdots & p_{n}
\end{array}\right]^{T}, n: \text { dimension of personality }
\end{aligned}
$$


Here, $P_{0}$ is a personality-emotion influence matrix that indicates how each personality factor influences each emotion.

Even though the same $\Psi_{\mathrm{e}}(\cdot)$ in the previous PME model was used, we employed a different internal updating function $\Omega_{\mathrm{e}}(\cdot)$. We used not a constant term but some functions for the internal updating to differentiate the amount of emotion that is internally updated according to emotion states. Vector $\mathbf{d}$ makes it possible.

$$
\begin{aligned}
& \Omega_{e}(\cdot)=\left[\begin{array}{c}
-C_{e} \\
\vdots \\
-C_{e}
\end{array}\right]_{m \times 1} \rightarrow \Omega_{e}(\cdot)=-C_{e} \cdot \mathbf{d} \cdot t^{2} \\
& \mathbf{d}=\left[\begin{array}{lll}
d_{1} & \cdots & d_{m}
\end{array}\right]^{T}, m: \text { dimension of emotion }
\end{aligned}
$$

Here, $C_{e}$ is a constant value, $\mathbf{d}$ is an emotion-decay vector, and $t$ is sampling time. So if (9) is used to internally update emotions, we are able to reflect different characteristics of each emotion's decay.

\section{Emotion Expression Model}

In chapter 3, we proposed an emotion generation model based on the PME model. Now, we will introduce an emotion expression model, LDAEM, and an integration of the expression model with the proposed emotion generation model.

\subsection{LDAEM}

Using LDAEM, we can easily get dynamic facial expressions according to the change of generated emotions. This model consists of the Linear Affect-Expression Space Model (LAESM) and the Dynamic Emotion Model (DEM), as in (10) and (11).

$$
\begin{gathered}
{ }^{r} \mathbf{p}=T \cdot \mathbf{e} \\
M \ddot{\mathbf{e}}+C \dot{\mathbf{e}}+K \mathbf{e}=\mathbf{s}
\end{gathered}
$$

In LAESM [9], affect space and expression space of a developed robot are defined first, and a relationship between the two spaces is determined. So, if an artificial emotion $\mathbf{e}$ is determined in the affect space, corresponding emotional expression ${ }^{\mathrm{r}} \mathbf{p}$ can be immediately manifested by (10). Here, $T$ is a transition matrix between affect space and expression space of the developed robot.

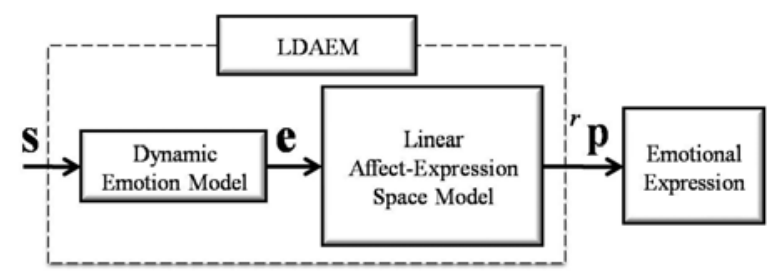

Fig. 4. Block diagram of LDAEM 
In DEM [10], trajectories of emotion e for input $\mathbf{s}$ are generated automatically considering characteristics of each emotion, as in (11). Here, $M, C$, and $K$ are the coefficient matrices that determine the characteristics of bases in the affect space.

\subsection{Integration between Emotion Generation Model and Emotion Expression Model}

To integrate the emotion expression model with the emotion generation model, all we have to do is use the generated emotions as inputs of DEM, as in (12).

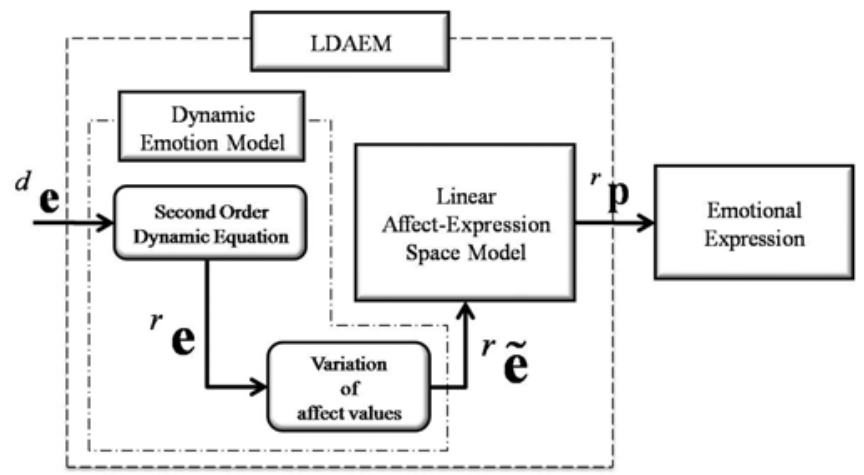

Fig. 5. Modified block diagram of LDAEM

Before applying the second order dynamic equation, we have to rectify the desired emotion ${ }^{\mathrm{d}} \mathbf{e}$ that is generated from the proposed emotion generation model. First, we multiplied the desired emotion ${ }^{\mathrm{d}} \mathbf{e}$ by matrix $E$, which represents a position of emotions in the affect space because the dimension of the desired emotion ${ }^{\mathrm{d}} \mathbf{e}$ and that of the current emotion ${ }^{\mathrm{r}} \mathbf{e}$ are different. Next, matrix $K$ has to be multiplied by the above result to minimize the steady-state error. Then, a trajectory between current emotion ${ }^{\mathrm{r}} \mathbf{e}$ and desired emotion ${ }^{\mathrm{d}} \mathbf{e}$ is determined through the second order differential equation.

$$
\begin{aligned}
& \mathbf{s}_{D_{e} \times 1}=K_{D_{e} \times D_{e}} \cdot E_{D_{e} \times m} \cdot{ }^{d} \mathbf{e}_{m \times 1} \\
& M \cdot \frac{d^{2}}{d t^{2}}{ }^{r} \mathbf{e}+C \cdot \frac{d}{d t}{ }^{r} \mathbf{e}+K \cdot{ }^{r} \mathbf{e}=\mathbf{s} \\
& D_{e}: \text { dimension of affect space } \\
& m: \text { number of generated emotions }
\end{aligned}
$$

To make more life-like facial expressions, we added a random value to the current emotion ${ }^{\mathrm{r}} \mathbf{e}$ in the affect space within an emotional boundary that was determined based on the recognition rate of facial expressions [11]. 


$$
\begin{aligned}
& { }^{r} \tilde{\mathbf{e}}=\left[\begin{array}{c}
\tilde{e}_{1} \\
\vdots \\
\tilde{e}_{D_{e}}
\end{array}\right]=\left\{\begin{array}{c}
{ }^{r} \mathbf{e}+\operatorname{rand}(\cdot), \quad\left\|{ }^{r} \mathbf{e}-{ }^{t} \mathbf{e}\right\| \leq{ }^{t} b \\
{ }^{r} \mathbf{e} \quad, \quad\left\|{ }^{r} \mathbf{e}-{ }^{t} \mathbf{e}\right\|>{ }^{t} b
\end{array}\right. \\
& { }^{t} \mathbf{e}=E \cdot{ }^{d} \mathbf{e},-1<\operatorname{rand}(\cdot)<1, \\
& { }^{r} \mathbf{e}: \text { current emotion vector, }{ }^{t} \mathbf{e}: t \arg \text { et emotion vector } \\
& { }^{r} \tilde{\mathbf{e}}: \text { newly generated emotion vector } \\
& { }^{t} b \text { : radius of emotional boundary }
\end{aligned}
$$$$
\text { for target emotion in the affect space }
$$

\section{Experimental Results}

In this chapter, the results of generated emotions and their corresponding facial expressions under various conditions will be shown. We used six kinds of external stimuli such as hit, sudden showing up, failure of tasks, pat, weird smell, and darkness as inputs of the emotion generation model; generated emotions are determined based on Ekman's six basic emotions: anger, surprise, sadness, happiness, disgust and fear. One dimensional mood (positive-negative) and five dimensional personality based on the Big Five Model were used.

Determination of each matrix used in the emotion generation model is very important. We might get absolutely different results according to the values of the four matrices. $P_{0}$ is suggested based on Julie's results [12] except for the emotion surprise. Surprise is generally considered as a reactive response, so it can be generated identically regardless of personality. Thus, we decided that the entries of $P_{0}$ related with the surprise emotion would be one. $Q$ was determined intuitively and the others were calculated as in (14).

$$
\begin{aligned}
& P_{0_{m \times n}}=\left[\begin{array}{ccccc}
0.04 & -0.01 & -0.01 & 0.01 & 0.12 \\
1.00 & 1.00 & 1.00 & 1.00 & 1.00 \\
-0.01 & 0.00 & -0.16 & 0.06 & 0.06 \\
0.18 & 0.23 & 0.29 & 0.23 & -0.16 \\
-0.17 & -0.12 & -0.24 & -0.04 & 0.24 \\
-0.24 & -0.32 & -0.22 & 0.06 & 0.38
\end{array}\right] \\
& Q_{k \times m}=\left[\begin{array}{llllll}
-0.3 & 0.0 & -0.1 & 0.3 & -0.2 & -0.15
\end{array}\right] \\
& R_{0_{k \times n}}=Q_{k \times m} \cdot P_{o_{m \times n}}=\left[\begin{array}{lllll}
0.113 & 0.144 & 0.187 & 0.059 & -0.195
\end{array}\right] \\
& T_{m \times k}=Q_{k \times m}{ }^{T}
\end{aligned}
$$

The first experiment looks at how differently mood is generated according to whether history of input and emotion are used or not. Here, we considered 50 previous input stimuli and emotion states. As shown in Fig. 6 (b), mood is changed more dynamically 
when only the current input stimuli are used than when history of input stimuli and emotions are used. For instance, a slop of mood's change in bottom graph of fig. 6 (b) is less gentle than that of the upper graph of fig. 6 (b), and mood in the bottom graph of fig. 6 (b) is not changed immediately according to user inputs.

In addition to the change of mood, patterns of generated emotions such as intensity and lasting time of emotion are changed a little because current mood participates in updating emotions.

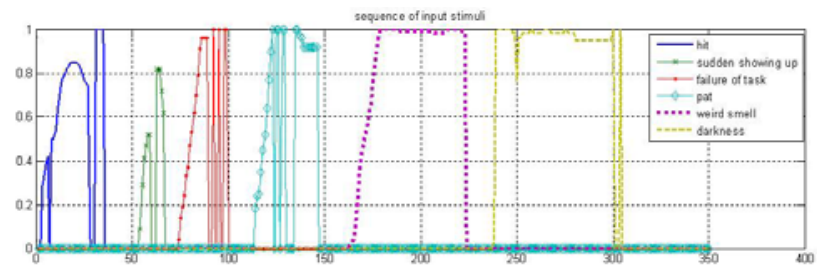

(a) A sequence of input stimuli
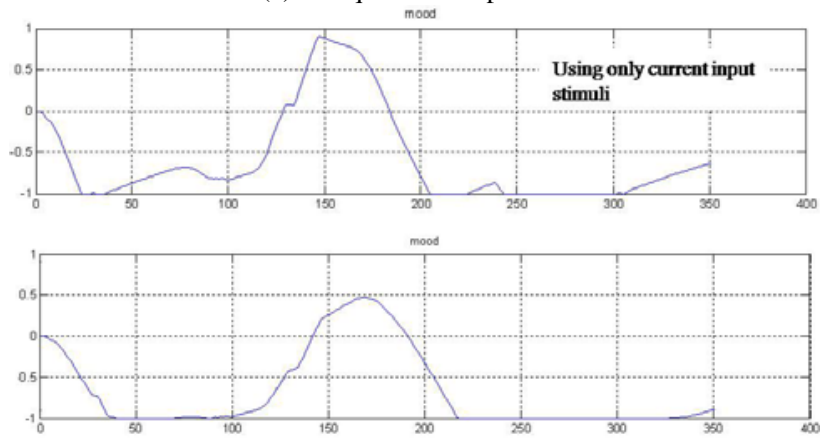

(b) A change of mood
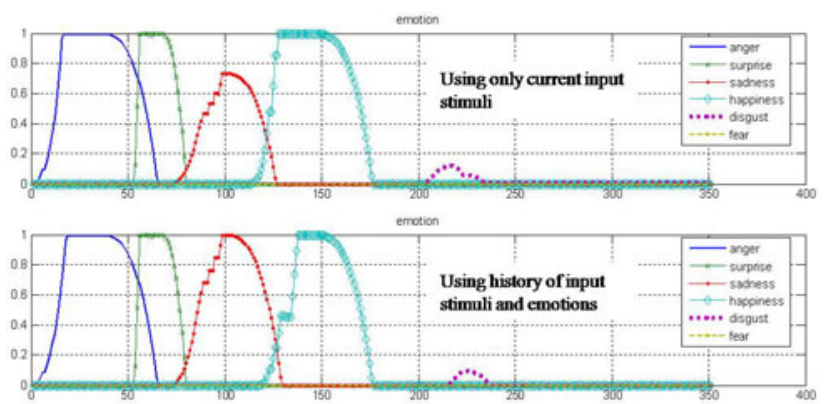

(c) A change of emotions

Fig. 6. A change of mood and emotions according to the using history of input stimuli and emotions

Second, we performed the experiment for two kinds of personality, such as strong openness and strong agreeableness to observe differences of the generated emotions. 
Elements of $\mathbf{p}$ in (15) are openness, conscientiousness, extraversion, agreeableness, and neurotic orderliness.

$$
\begin{aligned}
& \mathbf{p}=\left[\begin{array}{lllll}
1.0 & 0.1 & 0.1 & 0.1 & 0.1
\end{array}\right] \text { : strong openess } \\
& \mathbf{p}=\left[\begin{array}{lllll}
0.1 & 0.1 & 0.1 & 1.0 & 0.1
\end{array}\right] \text { : strong agreeableness }
\end{aligned}
$$

The sequence of input stimuli is the same as that shown in Fig. 6 (a). As shown in Fig. 7 (c), we found that generated emotions are completely different from each other according to each personality. In the case of agreeableness, all kinds of emotions were generated easily according to input stimuli. In the case of openness, however, sadness, disgust, and fear emotions were not generated as easily as they would be with an agreeable personality. This is due to the personality-emotion influence matrix $P_{0}$. In

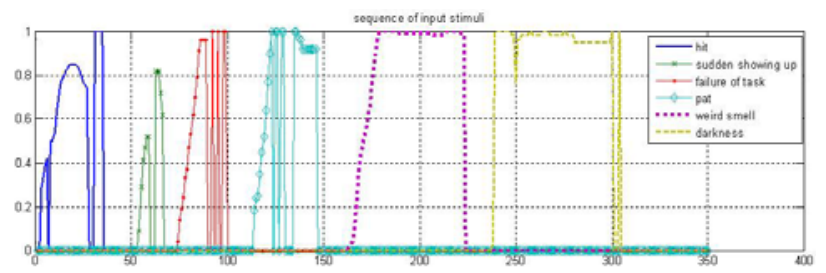

(a) A sequence of input stimuli
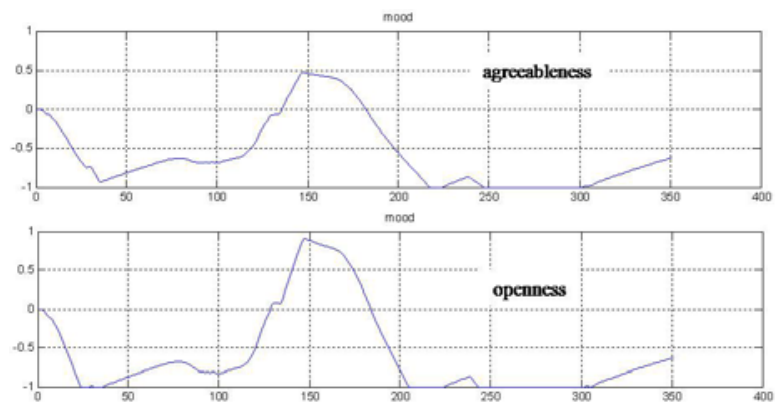

(b) A change of mood
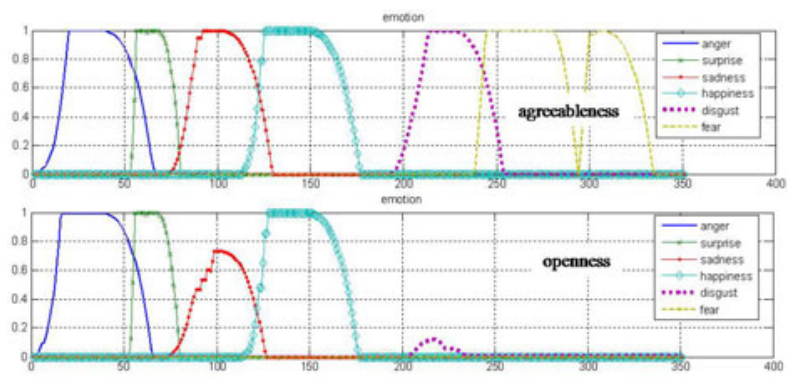

(c) A change of emotions

Fig. 7. A Change of mood and emotions according to personalities 
(14), the first column of $P_{0}$ is for the relationship between openness of personality and the six basic emotions, which are anger, surprise, sadness, happiness, disgust, and neurotic orderliness. The fourth column is for agreeableness of personality. Comparing the two columns, values of sadness, disgust, and fear for agreeableness are greater than those for openness. Therefore, the above three emotions for agreeableness were generated more easily than they were for the personality of openness. Furthermore, intensity of mood is quite dissimilar according to personalities because history of emotions participates in updating mood.

The final experiment is to check the change of the internal update of emotion according to each emotion. We can find that degree of internal update for surprise and happiness is relatively bigger than that value would be for other emotions.

Fig. 9 is a sequential change of facial expression using the results of Fig.8 as an input of proposed emotion expression model.

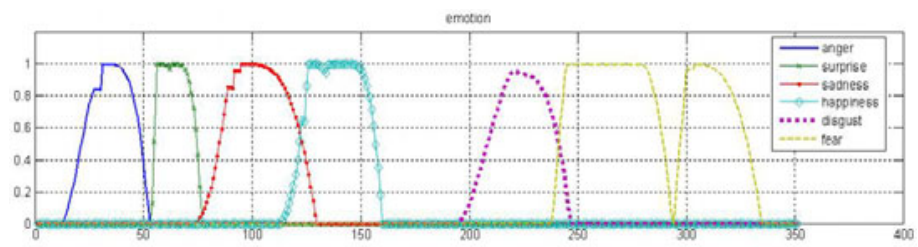

Fig. 8. Different internal update according to the kind of emotion

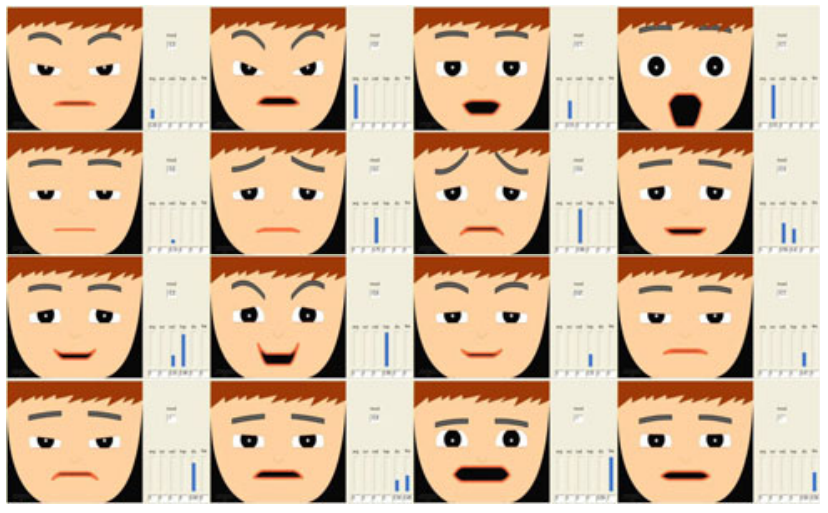

Fig. 9. A sequence of facial expressions using generated emotion

\section{Conclusion}

In this paper, we proposed an emotion generation model based on the PME model and a method to integrate the emotion generation model with the emotion expression model LDAEM. To implement the general characteristics of mood and emotion, we used a history of input and emotions. Through the experiments, we confirmed the differences between the previous methods and the proposed one. Furthermore, we suggested a guideline to determine the four matrices that were used in the PME 
model. Entries of those matrices are quite significant factors for generating emotions. We also enhanced the internal updating function of emotion. Finally, we integrated the proposed emotion generation model with LDAEM using matrix $E$, which represents a position of each emotion in the affect space.

Here we only updated emotions and mood, but the personality of robots also can be changed when a long term interaction between humans and robots is occurring. In this paper, we generated only six basic emotions, so this report might be considered slightly simple. So, we need to do more research about personality updating and need to expand the kinds of generated emotions.

\section{Acknowledgement}

This work was supported by the MKE(The Ministry of Knowledge Economy), Korea, under the ITRC(Information Technology Research Center) support program supervised by the IITA(Institute for Information Technology Advancement).

\section{References}

1. Breazeal, C.: Designing Social Robots. The MIT Press, Cambridge (2002)

2. Kshirsagar, S., Magnenat-Thalmann, N.: A Multilayer Personality Model. In: Proc. of 2nd International Symposium on Smart Graphics, pp. 107-115 (2002)

3. Egges, A., Kshirsagar, S., Magnenat-Thalmann, N.: Generic personality and emotion simulation for conversational agents. Computer Animation and Virtual Worlds 15(1), 1-13 (2004)

4. Park, J.C., Kim, H.R., Kim, Y.M., Kwon, D.S.: Robot's Individual Emotion Generation Model and Action Coloring According to the Robot's Personality. In: The 18th IEEE International Symposium on Robot and Human Interactive Communication Toyama, Japan, September 27-October 2 (2009)

5. Banik, S.C., Watanabe, K., Izumi, K.: Intelligent Behavior Generation of Benevolent Agents with a Stochastic Model of Emotion. In: Ishikawa, M., Doya, K., Miyamoto, H., Yamakawa, T. (eds.) ICONIP 2007, Part II. LNCS, vol. 4985, pp. 147-156. Springer, Heidelberg (2008)

6. Miwa, H., Itoh, K., Takanobu, H., Takanishi, A.: Development of Mental Model for Humanoid Robots. In: ROMANSY, 2004 Montreal, Canada, June 14-18 (2004)

7. Digman, J.M.: Personality structure: Emergence of the five-factor model. Annual Review of Psychology 41, 417-440 (1990)

8. Ekman, P., Friesen, W.V.: Unmasking the Face. Malor Books, Cambridge (2003)

9. Lee, H.S., Park, J.W., Chung, M.J.: A Linear Affect-Expression Space Model and Control Points for Mascot-Type Facial Robots. IEEE Transactions on Robotics 23(5) (October 2007)

10. Lee, H.S., Park, J.W., Jo, S.H., Chung, M.J.: A Linear Dynamic Affect-Expression Model: Facial Expressions According to Perceived Emotions in Mascot-Type Facial Robots. In: Proceedings 16th IEEE International Conference on Robot \& Human Interactive Communication, August 26 (2007)

11. Park, J.W., Kim, W.H., Lee, W.H., Kim, W.H., Chung, M.J.: Lifelike Facial Expression of Mascot-Type Robot based on Emotional Boundaries. In: 2009 IEEE International Conference on Robotics and Biomimetics, Guilin, Guangxi, China, December 19-23 (2009)

12. Penley, J.A., Tomaka, J.: Associations among the Big Five, emotional responses, and coping with acute stress. Personality and Individual Differences 32, 1215-1228 (2002) 